\title{
New necessary conditions for (negative) Latin square type partial difference sets in abelian groups
}

\author{
Zeying Wang * $\dagger$
}

\begin{abstract}
Partial difference sets (for short, PDSs) with parameters $\left(n^{2}, r(n-\epsilon), \epsilon n+r^{2}-\right.$ $\left.3 \epsilon r, r^{2}-\epsilon r\right)$ are called Latin square type (respectively negative Latin square type) PDSs if $\epsilon=1$ (respectively $\epsilon=-1$ ). In this paper, we will give restrictions on the parameter $r$ of a (negative) Latin square type partial difference set in an abelian group of non-prime power order. As far as we know no previous general restrictions on $r$ were known. Our restrictions are particularly useful when $a$ is much larger than $b$. As an application, we show that if there exists an abelian negative Latin square type PDS with parameter set $\left(9 p^{4 s}, r\left(3 p^{2 s}+1\right),-3 p^{2 s}+r^{2}+3 r, r^{2}+r\right)$, $1 \leq r \leq \frac{3 p^{2 s}-1}{2}, p \equiv 1(\bmod 4)$ a prime number and $s$ is an odd positive integer, then there are at most three possible values for $r$. For two of these three $r$ values, J. Polhill gave constructions in 2009 [8].
\end{abstract}

\section{Introduction}

Let $G$ be a finite abelian group of order $v$, and let $D \subseteq G$ be a subset of size $k$. We say $D$ is a $(v, k, \lambda, \mu)$-partial difference set (PDS) in $G$ if the expressions $g h^{-1}$, $g, h \in D, g \neq h$, represent each non-identity element in $D$ exactly $\lambda$ times, and each non-identity element of $G$ not in $D$ exactly $\mu$ times. If we further assume that $D^{(-1)}=D$ (where $D^{(s)}=\left\{g^{s}: g \in D\right\}$ ) and $e \notin D$ (where $e$ is the identity element of $G$ ), then $D$ is called a regular PDS. A regular PDS is called trivial if $D \cup\{e\}$ or

*Michigan Technological University

†zeying@mtu.edu 
$G \backslash D$ is a subgroup of $G$. The condition that $D$ be regular is not a very restrictive one, as $D^{(-1)}=D$ is automatically fulfilled whenever $\lambda \neq \mu$, and $D$ is a PDS if and only if $D \cup\{e\}$ is a PDS.

The Cayley graph over $G$ with connection set $D$, denoted by $\operatorname{Cay}(G, D)$, is the graph with the elements of $G$ as vertices, and in which two vertices $g$ and $h$ are adjacent if and only if $g h^{-1}$ belongs to $D$. When the connection set $D$ is a regular $\operatorname{PDS}$, Cay $(G, D)$ is a strongly regular graph. The importance of regular PDSs lies in the fact that they are equivalent to strongly regular graphs with a sharply transitive automorphism group.

In [6], S.L. Ma describes several families of PDSs. Among these are PDSs with parameters $\left(n^{2}, r(n-\epsilon), \epsilon n+r^{2}-3 \epsilon r, r^{2}-\epsilon r\right)$ for $\epsilon= \pm 1$. When $\epsilon=1$, the PDS is called a Latin square type partial difference set, and when $\epsilon=-1$, the PDS is called a negative Latin square type partial difference set. These types of PDSs have received a lot of attention, see for example [1, 2], 3], 7], 9].

Let $D$ be a regular $(v, k, \lambda, \mu)$-PDS, then $G \backslash D$ is a $(v, v-k, v-2 k+\mu, v-2 k+\lambda)$ PDS, and $(G \backslash D) \backslash\{e\}$ is a $(v, v-k-1, v-2 k-2+\mu, v-2 k+\lambda)$-PDS, and we call $(G \backslash D) \backslash\{e\}$ the complement of $D$. It is easy to verify that the complement of a (negative) Latin square PDS is again a (negative) Latin square type PDS (with the new parameter $r^{\prime}=n-r+\epsilon$ ). Thus when we study the existence of a (negative) Latin square type partial difference set (up to complements), we can add the restriction $1 \leq r \leq \frac{n+\epsilon}{2}$. As far as we are aware, no other general restrictions on the parameter $r$ are known.

In this article we will give restrictions on the parameter $r$ of Latin square type and negative Latin square type PDSs in abelian groups of order $a^{2} b^{2}$, where $\operatorname{gcd}(a, b)=1, a>1$, and $b$ is an odd positive integer $\geq 3$. Before stating our main result in the next section we provide an intuitive description of it here. As mentioned before, $r$ could theoretically take any value in the interval $\left[1, \frac{a b+\epsilon}{2}\right]$. We divide this interval in subintervals of length approximately $a$. We then show that within each of these subintervals $r$ can only take values in two subintervals of length approximately $b$. When $a$ is less than approximately $2 b$ this does not say anything, but when $a$ is much larger than $b$ this reduces the possible values $r$ can take significantly, as it reduces the number of possible values for $r$ to approximately $b^{2}$.

Throughout this paper, we will use the following standard notation: $\beta=\lambda-\mu$ and $\Delta=\beta^{2}+4(k-\mu)$.

\section{Main result and its proof}

Below we cite a result on "sub-partial difference sets" that was discovered in [5]. We quote this result in the form given in [6] (Theorem 7.1). 
Proposition 2.1 [5] Let $D$ be a nontrivial regular $(v, k, \lambda, \mu)-P D S$ in an abelian group $G$. Suppose $\Delta$ is a perfect square. Let $N$ be a subgroup of $G$ such that $\operatorname{gcd}(|N|,|G| /|N|)=1$ and $|G| /|N|$ is odd. Let

$$
\pi:=\operatorname{gcd}(|N|, \sqrt{\Delta}) \quad \text { and } \quad \theta:=\left\lfloor\frac{\beta+\pi}{2 \pi}\right\rfloor .
$$

Then $D_{1}=N \cap D$ is a (not necessarily non-trivial) regular $\left(v_{1}, k_{1}, \lambda_{1}, \mu_{1}\right)$-PDS in $N$ with

$$
v_{1}=|N|, \beta_{1}=\lambda_{1}-\mu_{1}=\beta-2 \theta \pi, \Delta_{1}=\beta_{1}^{2}+4\left(k_{1}-\mu_{1}\right)=\pi^{2},
$$

and

$$
k_{1}=|N \cap D|=\frac{1}{2}\left[|N|+\beta_{1} \pm \sqrt{\left(|N|+\beta_{1}\right)^{2}-\left(\Delta_{1}-\beta_{1}^{2}\right)(|N|-1)}\right] .
$$

Note: In Proposition 2.1, if $\Delta_{1}=|N|$, we have

$$
k_{1}=\frac{1}{2}\left[|N|+\beta_{1} \pm\left(\beta_{1}+1\right) \sqrt{|N|}\right] .
$$

Now we state our main theorem:

Theorem 2.2 Let $G$ be an abelian group of order $a^{2} b^{2}$, where $\operatorname{gcd}(a, b)=1, a>1$, and $b$ is an odd positive integer $\geq 3$. Let $D$ be an $\left(a^{2} b^{2}, r(a b-\epsilon), \epsilon a b+r^{2}-3 \epsilon r\right.$, $\left.r^{2}-\epsilon r\right)-P D S$ in $G$, where

$$
t \leq \frac{\epsilon a b-2 \epsilon r+a}{2 a}<t+1
$$

for some integer $t$. Then we have the following results:

(i) when $\epsilon=1$, that is, when $D$ is a Latin square type $P D S$, we have either

$$
\frac{(a+1) b(b+1-2 t)}{2(b+1)}-(b-1) \leq r \leq \frac{(a+1) b(b+1-2 t)}{2(b+1)}
$$

or

$$
\frac{(a-1) b(b-1-2 t)}{2(b-1)} \leq r \leq \frac{(a-1) b(b-1-2 t)}{2(b-1)}+(b+1) .
$$

(ii) when $\epsilon=-1$, that is, when $D$ is a negative Latin square type $P D S$, we have either

$$
\frac{(a+1) b(b-1+2 t)}{2(b-1)}-(b+1) \leq r \leq \frac{(a+1) b(b-1+2 t)}{2(b-1)}
$$

or

$$
\frac{(a-1) b(b+1+2 t)}{2(1+b)} \leq r \leq \frac{(a-1) b(1+b+2 t)}{2(1+b)}+(b-1) .
$$


Note that for fixed $t$ the condition $t \leq \frac{\epsilon a b-2 \epsilon r+a}{2 a}<t+1$ bounds $r$ to an interval of approximately length $a$, whereas the conclusion of the theorem bounds $r$ to two subintervals of approximately length $b$.

The proof of our main theorem is an application of Proposition 2.1 combined with a variance argument.

Proof of Theorem 2.2; As $D$ is an $\left(a^{2} b^{2}, r(a b-\epsilon), \epsilon a b+r^{2}-3 \epsilon r, r^{2}-\epsilon r\right)$-PDS in $G$, we have $\Delta=(\lambda-\mu)^{2}+4(k-\mu)=(\epsilon a b-2 \epsilon r)^{2}+4\left(r a b-r^{2}\right)=a^{2} b^{2}$. Let $N$ be a subgroup of $G$ of order $a^{2}$. By Proposition 2.1, we have $\pi=\operatorname{gcd}(|N|, \sqrt{\Delta})=a$, $\Delta_{1}=\pi^{2}=a^{2}=|N|$. Furthermore, $\beta_{1}=\beta-2 \theta \pi$, and $\theta=\left\lfloor\frac{\beta+\pi}{2 \pi}\right\rfloor$. When

$$
t \leq \frac{\beta+\pi}{2 \pi}=\frac{\epsilon a b-2 \epsilon r+\pi}{2 \pi}<t+1,
$$

we have $\theta=t$, and $\beta_{1}=\beta-2 t \pi=\epsilon a b-2 \epsilon r-2 t a$.

Since $\Delta_{1}=|N|$, by Proposition 2.1, we have

$$
\begin{aligned}
k_{1} & =|N \cap D|=\frac{1}{2}\left(|N|+\beta_{1} \pm\left(\beta_{1}+1\right) \sqrt{|N|}\right) \\
& =\frac{1}{2}\left(\left(a^{2}+\epsilon a b-2 \epsilon r-2 t a\right) \pm(\epsilon a b-2 \epsilon r-2 t a+1) a\right) .
\end{aligned}
$$

Assume that $G=N \times H$, where $H$ is a subgroup of $G$ of order $b^{2}$. Let $h_{1}, h_{2}$, $\cdots, h_{b^{2}-1}$ be the non-identity elements of $H$, let $\mathcal{B}_{h_{i}}=N h_{i} \cap D$, and $B_{i}=\left|\mathcal{B}_{h_{i}}\right|$, $i=1,2, \cdots, b^{2}-1$.

One easily sees that $x y^{-1}(x, y \in D, x \neq y)$ belongs to $N$ if and only if either both $x$ and $y$ belong to $N$ or both belong to $\mathcal{B}_{h}$ for some non-identity element $h \in H$. By double-counting, we have

$$
\sum_{i=1}^{b^{2}-1} B_{i}\left(B_{i}-1\right)+k_{1}\left(k_{1}-1\right)=k_{1} \lambda+\left(a^{2}-1-k_{1}\right) \mu .
$$

It is easy to see that $\sum B_{i}=k-k_{1}$. Combining this information with Equation (2), we obtain the following system of equations:

$$
\left\{\begin{aligned}
\sum B_{i} & =k-k_{1}, \\
\sum B_{i}^{2} & =k-k_{1}^{2}+k_{1}(\lambda-\mu)+\left(a^{2}-1\right) \mu .
\end{aligned}\right.
$$

Case 1: When $D$ is a Latin square type PDS, that is, when $\epsilon=1$, by (1) we have $k_{1}=\frac{1}{2}\left(\left(a^{2}+a b-2 r-2 t a\right) \pm(a b-2 r-2 t a+1) a\right)$. 
(i) If $\left.k_{1}=\frac{1}{2}\left(a^{2}+a b-2 r-2 t a\right)+(a b-2 r-2 t a+1) a\right)$, by (3) the variance equals

$$
\begin{aligned}
& \left(b^{2}-1\right) \sum B_{i}^{2}-\left(\sum B_{i}\right)^{2} \\
& =-\frac{1}{4} a^{2}\left(2+b+a b-b^{2}+a b^{2}-2 r-2 b r-2 b t-2 a b t\right) \\
& *\left(b+a b+b^{2}+a b^{2}-2 r-2 b r-2 b t-2 a b t\right),
\end{aligned}
$$

and hence is non-negative if and only if

$$
\frac{(a+1) b(b+1-2 t)}{2(b+1)}-(b-1) \leq r \leq \frac{(a+1) b(b+1-2 t)}{2(b+1)} .
$$

(ii) If $k_{1}=\frac{1}{2}\left[\left(a^{2}+a b-2 r-2 t a\right)-(a b-2 r-2 t a+1) a\right]$, by (3) the variance equals

$$
\begin{aligned}
& \left(b^{2}-1\right) \sum B_{i}^{2}-\left(\sum B_{i}\right)^{2} \\
& =-\frac{1}{4} a^{2}\left(b-a b-b^{2}+a b^{2}+2 r-2 b r+2 b t-2 a b t\right) \\
& *\left(-2+b-a b+b^{2}+a b^{2}+2 r-2 b r+2 b t-2 a b t\right),
\end{aligned}
$$

and hence is non-negative if and only if

$$
\frac{(a-1) b(b-1-2 t)}{2(b-1)} \leq r \leq \frac{(a-1) b(b-1-2 t)}{2(b-1)}+(b+1)
$$

Case 2: When $D$ is a negative Latin square type PDS, that is, when $\epsilon=-1$, by (1) we have $k_{1}=\frac{1}{2}\left(\left(a^{2}-a b+2 r-2 t a\right) \pm(-a b+2 r-2 t a+1) a\right)$.

(i) If $k_{1}=\frac{1}{2}\left[\left(a^{2}-a b+2 r-2 t a\right)+(-a b+2 r-2 t a+1) a\right]$, by (3) the variance equals

$$
\begin{aligned}
& \left(b^{2}-1\right) \sum B_{i}^{2}-\left(\sum B_{i}\right)^{2} \\
& =-\frac{1}{4} a^{2}\left(2-b-a b-b^{2}+a b^{2}+2 r-2 b r+2 b t+2 a b t\right) \\
& *\left(-b-a b+b^{2}+a b^{2}+2 r-2 b r+2 b t+2 a b t\right),
\end{aligned}
$$

and hence is non-negative if and only if

$$
\frac{b(1+a)(b-1+2 t)}{2(b-1)}-(b+1) \leq r \leq \frac{b(1+a)(b-1+2 t)}{2(b-1)} .
$$


(ii) If $k_{1}=\frac{1}{2}\left[\left(a^{2}-a b+2 r-2 t a\right)-(-a b+2 r-2 t a+1) a\right]$, by (3) the variance equals

$$
\begin{aligned}
& \left(b^{2}-1\right) \sum B_{i}^{2}-\left(\sum B_{i}\right)^{2} \\
& =-\frac{1}{4} a^{2}\left(-b+a b-b^{2}+a b^{2}-2 r-2 b r-2 b t+2 a b t\right) \\
& *\left(-2-b+a b+b^{2}+a b^{2}-2 r-2 b r-2 b t+2 a b t\right),
\end{aligned}
$$

and hence is non-negative if and only if

$$
\frac{b(-1+a)(1+b+2 t)}{2(1+b)} \leq r \leq \frac{b(-1+a)(1+b+2 t)}{2(1+b)}+(b-1) .
$$

\section{Application to Latin square type PDSs}

Letting $\epsilon=1$ and $t=(b-1) / 2$ in Theorem 2.2, we obtain the following Corollary:

Corollary 3.1 Let $G$ be an abelian group of order $a^{2} b^{2}, \operatorname{gcd}(a, b)=1, a>1$, and $b$ an odd positive integer $\geq 3$. Let $D$ be an $\left(a^{2} b^{2}, r(a b-1), a b+r^{2}-3 r, r^{2}-r\right)$-Latin square type $P D S$ in $G$ with $1 \leq r \leq a$. Then we have either

$$
1 \leq r \leq b+1
$$

or

$$
\frac{(a+1) b}{(b+1)}-(b-1) \leq r \leq \frac{(a+1) b}{(b+1)}
$$

When $b$ is the smallest prime power of $a b$, there are constructions satisfying the restriction $1 \leq r \leq b+1$ of Corollary [3.1, see [6]. We cite the result below:

Theorem 3.2 ([6]) Let $n=p_{1}^{a_{1}} p_{2}^{a_{2}} \cdots p_{s}^{a_{s}}$ where $p_{1}, p_{2}, \cdots, p_{s}$ are distinct primes. Then there exists an abelian regular $\left(n^{2}, r(n-1), n+r^{2}-3 r, r^{2}-r\right)-P D S$ whenever $1 \leq r \leq \min \left\{p_{i}^{a_{i}}+1\right\}$.

\section{Applications to negative Latin square type PDSs}

In this section, we will apply our main result to negative Latin square type PDSs. In particular, we will show that in an abelian group $G$ of order $9 m^{4}$, where $m$ is an odd 
positive integer and $\operatorname{gcd}(3, m)=1$, if there exists a negative Latin square type PDS with the parameter set $\left(9 m^{4}, r\left(3 m^{2}+1\right),-3 m^{2}+r^{2}+3 r, r^{2}+r\right)$ and $1 \leq r \leq \frac{3 m^{2}-1}{2}$, then there are at most 6 possible values for $r$. Furthermore, if $m=p^{s}$, where $p \equiv 1$ $(\bmod 4)$ is a prime number and $s$ is an odd positive integer, then there are at most 3 possible values for $r$. For two of these three $r$ values, Polhill gave constructions in 2009 [8]. Note that without our Main Theorem [2.2, the possible number of values $r$ could hypothetically take in such groups grows unboundedly as $m$ or $p$ grows.

Let $G$ be an abelian group of order $9 \mathrm{~m}^{4}$, where $m$ is an odd positive integer and $\operatorname{gcd}(3, m)=1$. Let $D$ be a $\left(9 m^{4}, r\left(3 m^{2}+1\right),-3 m^{2}+r^{2}+3 r, r^{2}+r\right)$ negative Latin square type PDS in $G$. Let $\epsilon=-1, t=-1, a=m^{2}$, and $b=3$ in Theorem 2.2, It follows that if $0 \leq r<m^{2}$, we have $\frac{3 m^{2}-3}{4} \leq r \leq \frac{3 m^{2}+5}{4}$. Let $\epsilon=-1, t=0$, $a=m^{2}$, and $b=3$ in Theorem 2.2. It follows that if $m^{2} \leq r<2 m^{2}$, then either $\frac{3 m^{2}-5}{2} \leq r \leq \frac{3 m^{2}+3}{2}$ or $\frac{3 m^{2}-3}{2} \leq r \leq \frac{3 m^{2}+1}{2}$.

In what follows we will need the following result of Leung, Ma, and Schmidt [4].

Theorem 4.1 (4]) Let $p$ be a prime and let $D$ be a regular PDS in an abelian group $G=H \times P$ where $P$ is a $p$-group and $|H|$ is not divisible by $p$. Suppose that the parameter $\Delta$ is a square. Let $h \in H$. Then

$$
|D \cap P h| \equiv \begin{cases}1 & (\bmod p-1) \text { if } h \in D \\ 0 & (\bmod p-1) \text { if } h \notin D\end{cases}
$$

Theorem 4.2 Let $G$ be an abelian group of order $9 p^{4 s}$, where $p$ is a prime number $\geq 5$, and $s$ is a positive integer. Then there does not exist a $\left(9 p^{4 s}, r\left(3 p^{2 s}+1\right),-3 p^{2 s}+\right.$ $\left.r^{2}+3 r, r^{2}+r\right)-P D S$ in $G$ with $r=\left(3 p^{2 s}-5\right) / 2$.

Proof: Assume by way of contradiction that $D$ is a $\left(9 p^{4 s}, r\left(3 p^{2 s}+1\right),-3 p^{2 s}+\right.$ $\left.r^{2}+3 r, r^{2}+r\right)$-PDS in $G$ with $r=\left(3 p^{2 s}-5\right) / 2$. Let $N$ be the subgroup of $G$ of order $p^{4 s}$. Applying Proposition 2.1 to the subgroup $N$, we obtain $\pi=p^{2 s}, \theta=0$, $\beta_{1}=-5$, and

$$
k_{1}=|N \cap D|=\frac{p^{4 s}-4 p^{2 s}-5}{2} \text { or } \frac{p^{4 s}+4 p^{2 s}-5}{2} .
$$

Let $G=N \times H$, where $H$ is the subgroup of $G$ of order 9. As in the proof of Theorem 2.2, let $h_{1}, h_{2}, \cdots, h_{8}$ be all non-identity elements of $H$, and let $\mathcal{B}_{h_{i}}=$ $N h_{i} \cap D, B_{i}=\left|\mathcal{B}_{h_{i}}\right|, i=1,2, \cdots, 8$. Now we discuss the existence of $D$ in two cases based on the possible size of $N \cap D$. 
(i) If $k_{1}=\left(p^{4 s}-4 p^{2 s}-5\right) / 2$, we use formula (3) from Theorem 2.2 and obtain that $8 \sum_{i=1}^{8} B_{i}^{2}-\left(\sum_{i=1}^{8} B_{i}\right)^{2}=0$. Hence we have

$$
B_{1}=B_{2}=\cdots=B_{8}=\frac{p^{2 s}\left(p^{2 s}-1\right)}{2} .
$$

According to Theorem 4.1, since $B_{i}=\frac{p^{2 s}\left(p^{2 s}-1\right)}{2} \equiv 0(\bmod p-1)$, we have $h_{i} \notin D, i=1,2 \cdots, 8$, that is, $D \cap H=\emptyset$. On the other hand, by applying Proposition 2.1 to the subgroup $H$, we see that $|D \cap H|=2$ or 8 (here $\pi=3$, $\theta=-1$, and $\beta_{1}=1$ ), a contradiction.

(ii) If $k_{1}=\left(p^{4 s}+4 p^{2 s}-5\right) / 2$, we have $8 \sum_{i=1}^{8} B_{i}^{2}-\left(\sum_{i=1}^{8} B_{i}\right)^{2}=-48 p^{4 s}<0$, thus this case will not occur.

Hence there does not exist a $\left(9 p^{4 s}, r\left(3 p^{2 s}+1\right),-3 p^{2 s}+r^{2}+3 r, r^{2}+r\right)$-PDS with $r=\left(3 p^{2 s}-5\right) / 2$.

Theorem 4.3 Let $G$ be an abelian group of order $9 p^{4 s}$, where $p$ is a prime number with $p \equiv 1(\bmod 4)$, and $s$ is an odd positive integer. Then there does not exist a $\left(9 p^{4 s}, r\left(3 p^{2 s}+1\right),-3 p^{2 s}+r^{2}+3 r, r^{2}+r\right)-P D S D$ in $G$ when $r=\left(3 p^{2 s}-3\right) / 4$ or $r=\left(3 p^{2 s}+5\right) / 4$.

Proof: We use the same notation as in the previous theorem. First assume $r=\left(3 p^{2 s}-3\right) / 4$.

Let $N$ be the subgroup of $G$ of order $p^{4 s}$. Applying Proposition 2.1 to the subgroup $N$, we have $\pi=p^{2 s}, \theta=-1, \beta_{1}=\left(p^{2 s}-3\right) / 2$, and

$$
k_{1}=|N \cap D|=\frac{3 p^{4 s}-3}{4} \text { or } \frac{p^{4 s}+2 p^{2 s}-3}{4} .
$$

Now we discuss the existence of $D$ in two cases based on the possible size of $N \cap D$.

(i) If $k_{1}=\left(3 p^{4 s}-3\right) / 4$, using formula (3) from Theorem 2.2, we obtain $\sum_{i=1}^{8} B_{i}^{2}=$ $-3 p^{4 s}\left(p^{2 s}-1\right) / 2<0$, thus this case will not occur.

(ii) If $k_{1}=\left(p^{4 s}+2 p^{2 s}-3\right) / 4$, we have $8 \sum B_{i}^{2}-\left(\sum B_{i}\right)^{2}=0$, and hence

$$
B_{1}=B_{2}=\cdots=B_{8}=\frac{p^{2 s}\left(p^{2 s}-1\right)}{4} .
$$

When $p \equiv 1(\bmod 4)$ and $s$ is an odd positive integer, we have

$$
B_{i}=\frac{p^{2 s}(p-1)\left(p^{2 s-1}+p^{2 s-2}+\cdots+p+1\right)}{4} \equiv \frac{(p-1)(2 s)}{4} \equiv \frac{p-1}{2} \not \equiv 0,1 \quad(\bmod p-1) .
$$

Clearly $B_{i} \not \equiv 0,1(\bmod p-1)$ contradicts the result of Theorem 4.1 
Hence there does not exist a $\left(9 p^{4 s}, r\left(3 p^{2 s}+1\right),-3 p^{2 s}+r^{2}+3 r, r^{2}+r\right)$-PDS with $r=\left(3 p^{2 s}-3\right) / 4$.

Next we assume that $r=\left(3 p^{2 s}+5\right) / 4$. As in the previous case, we apply Proposition 2.1 to the subgroup $N$. Then we get $\pi=p^{2 s}, \theta=-1, \beta_{1}=\left(p^{2 s}+5\right) / 2$, and

$$
k_{1}=\frac{3 p^{4 s}+8 p^{2 s}+5}{4} \text { or } \frac{p^{4 s}-6 p^{2 s}+5}{4} .
$$

(i) If $k_{1}=\left(3 p^{4 s}+8 p^{2 s}+5\right) / 4$, using formula (3) from Theorem 2.2, we have $\sum_{i=1}^{8} B_{i}^{2}=-p^{4 s}\left(3 p^{2 s}+5\right) / 2<0$, thus this case will not occur.

(ii) If $k_{1}=\left(p^{4 s}-6 p^{2 s}+5\right) / 4$, we have $8 \sum B_{i}^{2}-\left(\sum B_{i}\right)^{2}=0$, and hence

$$
B_{1}=B_{2}=\cdots=B_{8}=\frac{p^{2 s}\left(p^{2 s}+3\right)}{4} .
$$

When $p \equiv 1(\bmod 4)$ and $s$ is an odd positive integer, we have

$$
B_{i}=\frac{p^{2 s}(p-1)\left(p^{2 s}+\cdots+p+1\right)}{4}+p^{2 s} \equiv \frac{p-1}{2}+1 \not \equiv 0,1 \quad(\bmod p-1) .
$$

Clearly $B_{i} \not \equiv 0,1(\bmod p-1)$ contradicts the result of Theorem 4.1 ,

Hence there does not exist a $\left(9 p^{4 s}, r\left(3 p^{2 s}+1\right),-3 p^{2 s}+r^{2}+3 r, r^{2}+r\right)$-PDS with $r=\left(3 p^{2 s}+5\right) / 4$.

Now we summarize the results of this section in the following theorem:

Theorem 4.4 (i) Let $G$ be an abelian group of order $9 m^{4}$, where $m$ is a positive odd integer and $\operatorname{gcd}(3, m)=1$. If there exist a $\left(9 m^{4}, r\left(3 m^{2}+1\right),-3 m^{2}+r^{2}+\right.$ $\left.3 r, r^{2}+r\right)$ negative Latin square type PDS in $G$ with $r \leq \frac{3 m^{2}-1}{2}$, then

$$
r=\frac{3 m^{2}-3}{4}, \frac{3 m^{2}+1}{4}, \frac{3 m^{2}+5}{4}, \frac{3 m^{2}-5}{2}, \frac{3 m^{2}-3}{2} \text {, or } \frac{3 m^{2}-1}{2} .
$$

(ii) Furthermore, if $m=p^{s}$, where $p$ is a prime number with $p \equiv 1(\bmod 4)$, and $s$ is an odd positive integer, under the same assumption as in (i), we have

$$
r=\frac{3 p^{2 s}+1}{4}, \frac{3 p^{2 s}-1}{2}, \text { or } \frac{3 p^{2 s}-3}{2} .
$$


Here we want to mention that there are known constructions of negative Latin square type PDSs with some of the $r$ values from Theorem 4.4;

In 2010, Polhill [10] constructed Paley type partial difference sets (negative Latin square type PDS with $r=\frac{3 m^{2}-1}{2}$ ) in certain abelian groups of order $9 \mathrm{~m}^{4}$, where $m$ is a positive odd number $>1$. In 2009, Polhill [8] (Theorems 3.1 and 3.2) constructed negative Latin square type PDSs in $G^{\prime}=\mathbb{Z}_{3}^{2} \times \mathbb{Z}_{p}^{4 s}$, where $p$ is an odd prime, with parameters $\left(9 p^{4 s}, r\left(3 p^{2 s}+1\right),-3 p^{2 s}+r^{2}+3 r, r^{2}+r\right), r=\left(3 p^{2 s}-1\right) / 2$ and $r=\left(3 p^{2 s}-3\right) / 2$. Theorem 4.4 shows that Polhill's constructions cover most possible negative Latin square type PDS parameter sets in abelian groups of order $9 p^{4 s}$ when $p$ is a prime number with $p \equiv 1(\bmod 4)$, and $s$ is an odd positive integer.

Open problem: Does there exist a $\left(9 p^{4 s}, r\left(3 p^{2 s}+1\right),-3 p^{2 s}+r^{2}+3 r, r^{2}+r\right)$ PDS with $r=\left(3 p^{2 s}+1\right) / 4$ when $p$ is a prime number with $p \equiv 1(\bmod 4)$, and $s$ is an odd positive integer? We attempted to use the structural information one can derive from the proof of Theorem 2.2 to construct such PDS but did not succeed.

\section{References}

[1] R. A. Bailey and D. Jungnickel, Translation nets and fixed-point-free group automorphisms, J. Combin. Theory Ser. A, 55, 1-13, (1990)

[2] J. Davis and Q. Xiang, Negative Latin square type partial difference sets in nonelementary abelian 2-groups, Journal of London Math. Society, 70, 125-141, (2004)

[3] J. Davis and Q. Xiang, Amorphic association schemes with negative Latin square-type graphs, Finite Fields Appl., 12, 595-612, (2006)

[4] K.H. Leung, S.L. Ma, B. Schmidt, Proper partial geometries with Singer groups and pseudogeometric partial difference sets, Journal of Combinatorial Theory (A), $115,147-177$ (2008)

[5] S. L. Ma, On subsets of partial difference sets, Discrete Mathematics 125, 263$272(1994)$

[6] S.L. Ma, A survey of partial difference sets, Designs, Codes, Cryptogr. 4, 221-261 (1994)

[7] J. Polhill, New negative Latin square type partial difference sets in nonelementary abelian 2-groups and 3-groups, Des. Codes Cryptogr., 46, 365-377, (2008)

[8] J. Polhill, Paley type partial difference sets in non p-groups, Designs, Codes and Cryptogr. 52, 163-169 (2009) 
[9] J. Polhill, Negative Latin square type partial difference sets and amorphic association schemes with Galois rings, J. Combin. Des., 17, 266-282, (2009)

[10] J. Polhill, Paley partial difference sets in groups of order $n^{4}$ and $9 n^{4}$ for any odd $n>1$, Journal of Combinatorial Theory (A), 117, 1027-1036, (2010) 